3.

Biblische und theologische Weichenstellungen 


\section{„Der Gerechte kennt das Bedürfnis seines Viehs.“ (Spr 12,10 a) Ethische Aspekte des Mensch-Tier-Verhältnisses im Alten Testament}

Ute Neumann-Gorsolke

\section{Hinführung: Die Rückkehr des Wolfes}

Der Wolf galt seit 1850 in Deutschland als ausgestorben bzw. ausgerottet, doch seit Ende des letzten Jahrhunderts gibt es eine Einwanderung aus den baltischen Staaten über Polen und auch aus Dänemark. Inzwischen leben 73 Rudel, 30 Paare und drei residente Einzeltiere in Deutschland und sind unter strengen Naturschutz gestellt. Von Naturschützern und Tierfreunden bejubelt ${ }^{1}$, sind die Schafzüchter und Landwirte alarmiert und fordern staatlichen Schutz ihrer Herden. Insbesondere Fälle, in denen Wölfe die Schutzzäune überwinden und Schafe reißen, haben die öffentliche Diskussion angeheizt. ${ }^{2}$ Während die einen den Abschuss dieser „Problemwölfe“ fordern, lehnen Naturschutzverbände und Organisationen wie der „Freundeskreis freilebender Wölfe" diese Maßnahmen ab. Die Fronten sind verhärtet.

Auf der Homepage des Bundes für Umwelt und Naturschutz Deutschland (BUND) findet sich folgender Text:

„Trotz seiner stark landwirtschaftlich geprägten Natur ist das Land (gemeint ist Schleswig-Holstein, U. N.-G.) auch ein Lebensraum für unzählige Wildtiere, die neben und mit dem Menschen und seinen Hausund Nutztieren leben. Auch Wölfe können hier eine Nische finden. Wenn wir sie lassen. Wir würden damit einen kleinen Beitrag zu Wie-

1 Vgl. die Homepage des Naturschutzbund-Deutschland (NABU): Wölfe in Deutschland. Ein Wildtier kehrt zurück, in: www.nabu.de/tiere-und-pflanzen/saeugetiere/ wolf/index.html (abgerufen am 02.09.2019).

2 In Norddeutschland, in meinem Heimatkreis Pinneberg und in den angrenzenden Kreisen ist es in den letzten Monaten zu zahlreichen „Wolfsüberfällen“ gekommen: Ein Wolf mit der Kennzeichnung GW 924 m hatte Schutzzäune überwunden und über 20 Schafe gerissen. Im Januar 2019 wurde dieser „Problemwolf“ nun vom Umweltministerium zum Abschuss frei gegeben. Bislang konnte der Wolf jedoch nicht „entnommen“ werden. 
derherstellung natürlicher Prozesse in unseren heimischen Ökosystemen leisten. Der Wolf ist Teil der natürlichen Landschaft SchleswigHolsteins, die die menschliche Kultur in einem überwältigenden Großteil in Nutzung genommen hat. Einen Nutzen für den Menschen zu erfüllen, ist aber nicht Voraussetzung, damit Tier-oder Pflanzenarten in der Landschaft, die wir mit ihnen teilen, existieren, dürfen'. Nicht der Wolf muss also begründen warum er dort existiert, sondern die, Wolfsgegner' müssen begründen welches Recht sie haben ihm sein Existenzrecht abzuerkennen. Es ist deshalb nicht nur ein Gebot des Naturschutzes, sondern auch der menschlichen Ethik dem Wolf dieses Lebensrecht zuzugestehen. Dies wäre für den Menschen kaum eine Einschränkung, für die Natur, die Ökosysteme und die betroffenen Tiere aber ein großer Gewinn“" (www.bund-sh.de/wolf/; abgerufen am 02.09.2019). ${ }^{3}$

Deutlich wird: Die Rückkehr des Wolfes provoziert aktuell „tier"ethische Fragen, die eben zuvörderst Fragen menschlicher Ethik sind: Hängt das Existenzrecht von Tieren am Nutzen des Menschen? Mit welchem Recht dürfen Menschen Tieren ihr Existenzrecht aberkennen? Dürfen sie es überhaupt?

Wenn auch der mediale Hype um den Wolf Fragen wie diese in das öffentliche Bewusstsein heben, so sind sie doch jenseits der "Problemwölfe“ längst von existentieller Bedeutung, nicht nur für die Tiere selbst, sondern auch für uns Menschen. Das Artensterben in Wald und Feld, in der Luft und im Meer führt uns drastisch vor Augen, dass unsere Art zu leben im wahrsten Sinne des Wortes tödlich ist: zuerst für die Bienen und dann für uns selbst!

So muss auch unsere Lebensart und d. h. auch der Umgang mit den domestizierten Tieren in den Blick genommen werden - und ich meine nicht die Herzenslieblinge Hund, Katze, Hamster -, sondern die dem modernen Menschen fernen „Haus-Tiere“, die unsere Nahrungsbasis darstellen. Denn trotz aufrüttelnder Berichte über „geschredderte“ männliche Küken und erschlagene schwächliche Ferkel, Legebatterien und abstoßender Bilder von Massentierhaltung bestimmen immer noch Fleischproduktion (!) und -verzehr unseren alltäglichen Umgang mit Huhn, Rind, Schwein und Lamm - eben als abgepackte Ware in der Fleischtheke. Wir leben zutiefst entfremdet selbst von der uns umgebenden domestizierten Tierwelt, ein Umstand, der im tierethischen Diskurs bedacht sein will.

3 Die fehlenden Kommata wurden nicht ersetzt. 
Wie und woher können in der heutigen Situation plausible Denkmodelle für unseren (zukünftigen) Umgang mit den Tieren, den domestizierten und den wild lebenden, erarbeitet werden? Welche Grundlagen bestimmen die Haltung der Christen, die sie in den ethischen Diskurs einbringen können? Und: Im Hinblick auf die Bedeutung der alttestamentlichen Schriften im sozialethischen Diskurs: Wie können die vielfältigen Aussagen der alttestamentlichen Überlieferung ${ }^{4}$ Grundlage für eine christliche Tierethik sein? Welche Impulse vermag der erste Teil der christlichen Bibel einzubringen?

Ich möchte hier Überlegungen von Rainer Kessler aufnehmen, die er in seiner 2017 erschienenen Ethik des Alten Testaments mit dem Titel Der Weg zum Leben eingebracht hat.

Er verweist mit Hans Joas auf „die historische Entstehung allen Glaubens, aller Geltungsansprüche auf Wahrheit und Werthaftigkeit" (Rainer Kessler 2017, 572). Ein modernes christliches Bewusstsein könne daher nicht auf den biblischen Bezug verzichten, sonst würde es sich „vom wesentlichen Entstehungsgrund seines Denkens und Handelns abschneiden“ (Rainer Kessler 2017, 573). Gerade in ihrer Fremdheit und ihrem Anderssein seien die biblischen Texte ein wichtiger Widerpart, der die vermeintlichen Selbstverständlichkeiten gegenwärtigen Denkens und darauf beruhenden Handelns im wörtlichen Sinne, in Frage stelle', ohne dass notwendigerweise die richtigen Antworten für heute bereitgehalten werden (vgl. Rainer Kessler 2017, 573).

Mit meinem Beitrag möchte ich den Weg für diesen „Suchprozess in Auseinandersetzung mit den historisch abständigen Texten“ (Rainer Kessler 2017, 573) bereiten und exemplarisch wichtige Texte der hebräischen Bibel zum Mensch-Tier-Verhältnis beleuchten.

Zunächst bedarf es einiger sozialgeschichtlicher Überlegungen zum Mensch-Tier-Verhältnis im alten Israel, das viele Texte des Alten Testaments mitprägt.

\section{Das Tier in der Lebenswelt des alten Israel}

Tiere sind in der Lebenswelt des alten Israel „allgegenwärtig“ (vgl. Othmar Keel 1993, 155-193). Über 200 Begriffe für Tiere weisen die hebräischen

4 Das Alte Testament als Schriftensammlung bietet keine kohärente Ethik, sondern zeigt in seinen unterschiedlichen Schriften eine Vielfalt von ethischen Positionen, die eng an die jeweilige politisch-soziale Situation gebunden sind. 
Texte der Bibel auf, vom Floh, der Motte und Ameise bis hin zum Geier, Bären, Hirschen, Löwen und Panther. ${ }^{5}$ Und auch ikonographische Zeugnisse der Levante (s. Abb. 1 und 2) führen uns vor Augen, wie vielfältig und artenreich die Fauna des antiken Palästina/Israel war. ${ }^{6}$

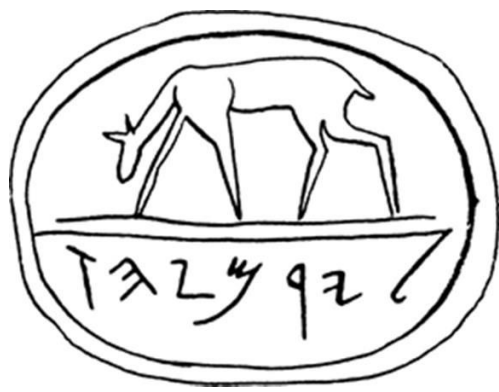

Abb. 1: Zweiregistriges Stempelsiegel aus Juda (8. Jh. v. Chr.) zeigt eine äsende Hirschkuh; darunter der Name des Besitzers: Dem Yirmeyahu (gehörig).

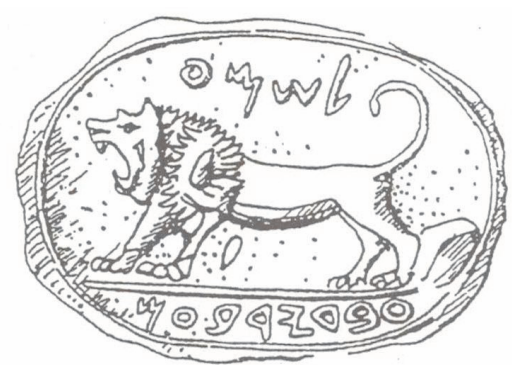

Abb. 2: Zweiregistriges Stempelsiegel aus Megiddo (8. Jh. v. Chr.) zeigt einen brüllenden Löwen mit erhobenem Schwanz. Die Inschrift nennt den Besitzer des Siegels „Schema, Diener [Minister] Jerobeams (II)“.

Für das Verhältnis Mensch-Tier ist im Alten Testament eine kategoriale Unterscheidung leitend, die in der tierethischen Diskussion bisweilen schon als anthropozentrisch kritisiert wird (vgl. Friederike Schmitz 2014, 13-76): die Unterscheidung in wild lebende Tiere (hebr. hajjat haśädaeh) und Haus- und Nutztiere (hebr. behemāh).

Wild lebende Tiere stellten für die Menschen „häufig eine ernstzunehmende Gefahr dar, auch im Alltagsleben. Der Mensch war durchaus nicht immer das überlegene Gegenüber" (Peter Riede 2002 a, 29). Anschaulich setzt diese Gefährdung des Menschen durch Wildtiere der Weheruf Am 5,18-20 in Szene, der jene verdammt, die den Tag JHWHs als lichten Freudentag erwarten:

18 Wehe denen, die den Tag JHWHs herbeisehnen! Wozu soll euch denn der Tag JHWHs sein? Er wird Finsternis sein und nicht Licht:

5 Die meisten Großtiere sind heute - wie bei uns - ausgestorben oder wurden ausgerottet. Löwen etwa sind nur bis zum 13. Jh. n. Chr. in Palästina nachweisbar, in Syrien bis ins 19. Jh., im Iran und Irak sogar bis ins erste Drittel des 20. Jh., vgl. Othmar Keel et al. 1984, 143.

6 Wie archäologische Funde zeigen, war bis ins 10. Jh. v. Chr. sogar das Flusspferd am See Genezareth heimisch, vgl. Iris Thomsen 2012. 
${ }^{19}$ Wie wenn jemand vor dem Löwen flieht, und es begegnet ihm der $B \ddot{r}$, aber er kommt nach Hause und stützt seine Hand auf die Mauer, da beißt ihn die Schlange.

${ }^{20}$ Wird nicht der Tag JHWHs Finsternis sein und nicht Licht? Dunkelheit und nicht Glanz ist ihm (eigen). ${ }^{7}$

Doch Löwe und Bär bedrohten nicht nur Leib und Leben der Menschen ${ }^{8}$, sie waren auch eine Gefahr für die Kleinviehherden der Menschen, wie sich aus der Rede des jungen Hirten David in 1 Sam 17,34-36 erschließen lässt:

${ }^{34}$ Und David sagte zu Saul: Dein Diener hat für seinen Vater die Schafe gehütet. Und wenn ein Löwe kam oder ein Bär und ein Schaf von der Herde nahm,

35 so zog ich aus, ihm nach, erschlug ihn und riss es ihm aus dem Maul. Erhob er sich aber gegen mich, so ergriff ich ihn beim Bart, schlug ihn und tötete ihn.

${ }^{36}$ Sowohl den Löwen als auch den Bären hat dein Diener erschlagen [...].

Da überdies wilde Tiere Gegenden bevölkerten, die für den Menschen unwirtlich und lebensfeindlich waren - wie Ruinen und wüste Landstriche -, werden sie in Unheilsansagen der Propheten in die Nähe von Dämonen gerückt. Als ein Beispiel sei hier ein Gerichtswort Jesajas über Edom angeführt:

${ }^{8}$ Denn es ist ein Tag der Rache JHWHs, ein Jahr der Vergeltungen für den Streit Zions.

${ }^{9}$ Und seine Flusstäler verwandeln sich in Pech und sein Staub in Schwefel, und sein Land wird zu brennendem Pech.

${ }^{10} \mathrm{Nacht}$ und Tag wird es nicht erlöschen, sein Rauch steigt auf für immer, von Generation zu Generation wird es in Trümmern liegen, niemand wird es durchstreifen.

${ }^{11}$ Und Eule und Igel nehmen es in Besitz, und Ibis und Rabe hausen darin, und die Messschnur der Verödung spannt er darüber aus und das Senkblei der Leere.

7 Übersetzung der biblischen Texte, falls nicht anders angegeben, durch die Autorin U. N.-G.

8 Mehrfach berichtet das Alte Testament, dass JHWH Löwen als Strafmittel gegen sein Volk einsetzt, vgl. 2 Kön 17,25 f. 
${ }^{12}$ Seine Edlen, aber niemand ist dort, der das Königtum ausruft, und alle seine Fürsten sind am Ende.

${ }^{13}$ Und in seinen Palästen werden Dornen aufsprießen, Unkraut und Dornensträucher in seinen befestigten Städten, und es wird eine Wohnstatt für Schakale sein, eine Wiese für junge Strauße.

${ }^{14}$ Und Wüstentiere werden auf Wildhunde treffen, und Bocksgeister begegnen einander, nur Lilit kann dort noch verweilen und eine Stätte der Ruhe finden für sich.

${ }^{15}$ Dort baut die Schlange ihr Nest und legt Eier und brütet sie in ihrem Schatten aus, dort können nur Raubvögel sich noch versammeln und sich ihr Stelldichein geben. (Jes 34,8.11-15)

Während Wildtiere dem Menschen in seiner Lebenswelt vielfach als feindlich erschienen und als bedrohlich erlebt wurden ${ }^{9}$ - und das schließt nicht aus, dass die Kraft und Macht der Wildtiere wie Löwe und Wildstier nicht auch ein Faszinosum waren und vielfältig Eingang in die Bildsprache erhielten (vgl. Peter Riede 2010) -, war das Verhältnis zu den Haus- und Nutztieren von anderer Art. Als Nutz- und Arbeitstiere bildeten Schafe (Fettschwanzschafe) und Ziegen, das so genannte Kleinvieh (hebr. șōn), bisweilen Rinder (Großvieh), Esel und Tauben die Lebensgrundlage in weiten Bevölkerungsteilen. Während die halbnomadisch lebenden Erzeltern wie Abraham und Sarah mit ihren Familien und ihrem Kleinvieh im Land umherzogen und zeitweilig im Schutz befestigter Städte lebten, wurde das Vieh der Ortschaften von Hirten, oft die jüngeren Familienangehörigen wie z. B. David, außerhalb gehütet und bewacht. In den Ortschaften lebten Esel, einige Ziegen und Tauben unter einem Dach mit den Menschen und bildeten geradezu eine Hausgemeinschaft (vgl. 2 Sam 12). Die spezielle Architektur des sog. Vierraumhauses (vgl. Shlomo Bunimovitz/ Avraham Faust 2009) war dazu besonders geeignet (s. Abb. 3):

9 Im Alten Testament werden Jagden trotz des Wildreichtums selten explizit genannt. Dabei umfasste die Auflistung reiner Tiere, die den Israeliten als Speise erlaubt waren, durchaus „Hirsch, Gazelle, Damhirsch, Wildziege und alle Arten von Antilopen“ (Dtn 14,5; vgl. auch 12,15). Wiederum erwähnt nur 1 Kön 5,3, dass Wildbret wie Gazelle und Karmelreh die üppige königliche Tafel ergänzten. Auch viele Bildworte vor allem der Psalmen und in der Weisheitsliteratur lassen aber darauf schließen, dass mit Netzen oder Klappnetzen Vögel gefangen oder sie mit einem Wurfholz getötet wurden. Dazu werden Schleppnetz, Pfeil und Bogen und Angel genannt, die für die Jagd von Wildstieren, Gazellen und Fischen genutzt worden sein dürften. Allerdings verherrlicht die Bildsprache der Psalmen die Jagd nicht, sondern stellt die Erfahrung des Gejagten in den Mittelpunkt, vgl. Peter Riede 2000 . 


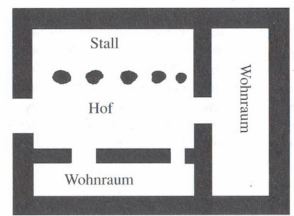

$0 \longdiv { 1 2 3 4 5 }$

Abb. 3: Grundriss eines eisenzeitlichen Vierraumhauses, in dem Menschen und Tiere zusammen lebten; im nicht überdachten Hof spielte sich das Arbeitsleben ab.

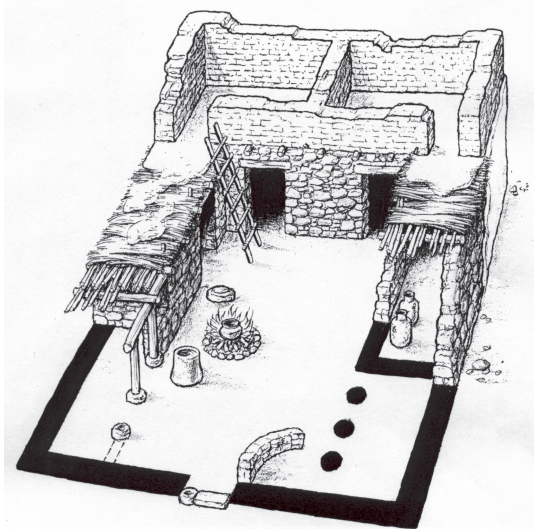

Abb. 4: Rekonstruktion eines eisenzeitlichen Vierraumhauses.

Schafe und Ziegen waren die Existenzgrundlage des täglichen Lebens. Sie lieferten den Menschen Milch, aus der auch Butter gemacht wurde, Wolle, Leder und Felle. Das Fleisch der Tiere wurde dagegen selten gegessen; wenn eines der kostbaren Tiere geschlachtet wurde, dann für ein Fest (vgl. Lk 15,11-31) oder einen besonderen Gast (vgl. Gen 18) oder für den Opferkult am Tempel. ${ }^{10}$

Darüber hinaus waren diese Tiere, vor allem Rinder und Esel, auch als Arbeitstiere unerlässlich bei der mühevollen Feldarbeit: Während die Rinder einzeln oder in Paaren - nie aber zusammen mit anderen Tieren (vgl. Dtn 22,10) - den sog. Hakenpflug zogen, um die Erde aufzulockern, oder den sog. Dreschschlitten, um das Korn zu dreschen, fungierten Esel als Lasttiere und trugen bei der Ernte die Garben auf die Tenne.

Die gesamte Existenz der bäuerlichen Großfamilien hing - das machen diese wenigen Ausführungen deutlich - an diesen Tieren und ihrem Ge-

10 Vor diesem Hintergrund wird auch der Wert eines Tieropfers deutlich, das z. B. für die Auslösung der Erstgeburt, bei Wiedergutmachungen oder bei Festritualen gegeben wurde. Vgl. auch Bernd Janowski 1999, 40, der betont, dass Tieropfer „in antiken Gesellschaften immer Verzicht (bedeutet), weil das geopferte Tier als Gabe an die Gottheit (zumindest teilweise) dem unmittelbaren Verzehr entzogen wird. Der Mensch gibt mit dem Opfertier etwas her, dessen er selber dringend bedarf“. 
deihen (vgl. Ps 144,12-14). Es nimmt daher nicht wunder, dass in weisheitlichen Texten und vor allem alten Rechtsbestimmungen, die dezidiert auf die Lebenswirklichkeit der Menschen ausgerichtet sind, das Verhältnis Mensch-Tier in den Blick rückt. Diese Texte sollen jetzt betrachtet werden.

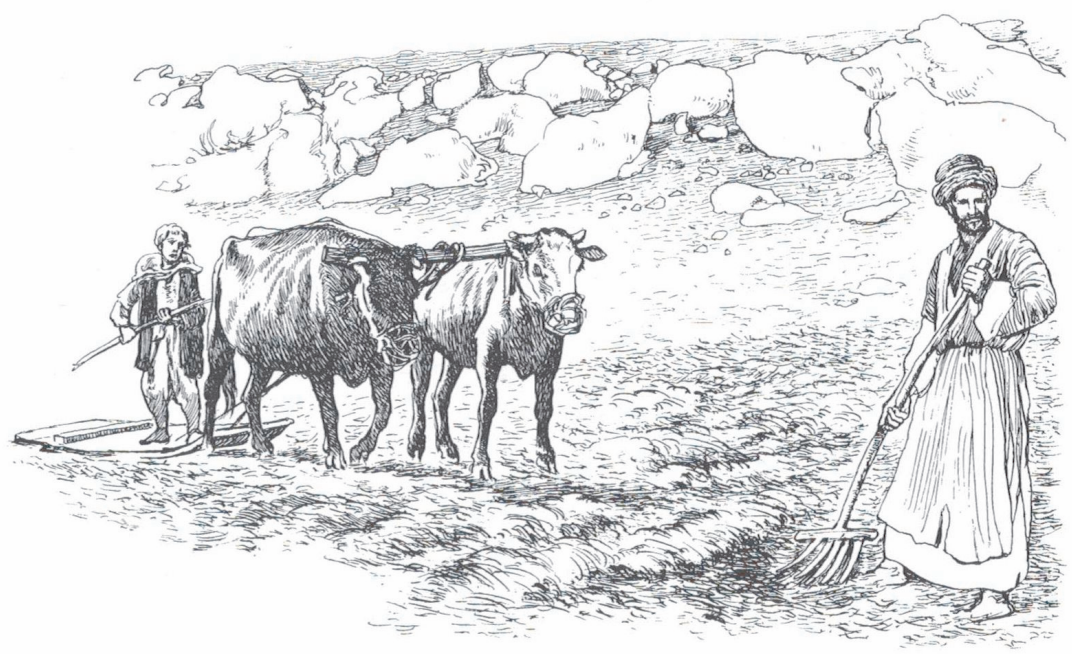

Abb. 5: Diese Zeichnung gibt den Dreschvorgang im 20. Jahrhundert n. Chr. wieder, entspricht aber der alttestamentlichen Zeit: Rinder zogen einen einfachen Dreschschlitten, der tagelang über das geerntete Getreide fuhr: Durch die an der Unterseite des Dreschschlittens eingelassenen Flintsteine lösten sich allmählich die Körner aus den Ähren.

\section{Mensch und Tier in der Weisheit und in Rechtstexten}

\subsection{Weisheitliche Texte}

Die älteren Weisheitsschriften des Alten Testaments gründen in der lebensnahen Weisheit der bäuerlichen Großfamilie. Es geht ihr nicht um philosophische Diskurse, sondern alltägliches Sozialverhalten: um „durch Praxis gewonnenes und auf Praxis zielendes Alltags- und Erfahrungswissen" (Erich Zenger/Christian Frevel 2012, 405), dessen Ziel gelingendes Leben ist - nicht nur des eigenen, sondern auch der Familie und des Sippenverbandes. Getragen wird diese lebensnahe Weisheit von der Überzeu- 
gung, „dass die Welt in geordneten Zusammenhängen steht, in denen alles mit allem in Verbindung steht" (Erich Zenger/Christian Frevel 2012, 406) und jedes Handeln Folgen hat. ${ }^{11}$ Diese Zusammenhänge gilt es zu erkennen und $\mathrm{zu}$ beherzigen. M. Görg nannte die Ältere Weisheit deshalb „Theologie der praktischen Vernunft" (zitiert bei Erich Zenger/Christian Frevel 2012, 407). Daher dominiert u. a. das Mahnwort als fiktives LehrerSchüler-Wort im Proverbienbuch (Spr). Ein Beispiel stellt Spr 27,23-27 dar, das zur Sorge um das Kleinvieh mahnt:

${ }^{23}$ Kümmere dich sorgfältig (hebr. Verb jäd $\bar{a}^{-}$) um das Aussehen deines Kleinviehs, richte deine Aufmerksamkeit (wörtl.: richte dein Herz) auf die Herden!

${ }^{24}$ Denn nicht bis in ferne Zeiten (reicht) der Vorrat. Und (währt) etwa eine Krone von Generation zu Generation?

${ }^{25}$ Ist das Heu geerntet und erscheint frisches Gras und sind eingesammelt die Kräuter der Berge,

26 (dann gibt es) Lämmer zu deiner Bekleidung und Böcke als Kaufpreis für ein Feld;

27 und genug Ziegenmilch ist da zu deiner Nahrung, zur Nahrung deines Hauses, und Lebensunterhalt für deine Mägde.

Auf die einleitende doppelte Mahnung „kümmere dich sorgfältig/richte deine Aufmerksamkeit" folgt in V. 24 die Begründung: Kein Vorrat währt ewig! und in V. 25-27 wird dem positiver Lohn in Aussicht gestellt, der sich an die Mahnung in V. 23 hält: Bekleidung durch die Lämmer; Böcke als Kaufpreis für Land und Ziegenmilch als Grundlage der Ernährung. Die Mahnung in V. 23 ist von großer Eindringlichkeit, die grammatisch durch die figura etymologica von jäd $\bar{a}$ ' betont wird, d. h., dass das Verb doppelt, sowohl in flektierter Form als auch als Infinitiv absolutus, erscheint. Das Verb jā $\bar{a} \bar{a}^{\epsilon}$ bezeichnet sowohl Erkenntnis und Wissen und meint zudem „das praktische, emotionale und volitive ,Kennenlernen“" (Gerhard J. Botterweck 1982, 494): das Sich-Kümmern, Achtgeben. Dem entspricht ebenfalls die Aufforderung, das Herz den Herden zuzuwenden. Hebr. leb ,Herz ist das zentrale menschliche Organ des Wissens und Entscheidens. ${ }^{12}$ Auf der semantischen Ebene sind Erkennen/Wissen und das sich daraus ergebende Handeln, die Fürsorge, in eins gesetzt: Wer von den Tieren gut le-

11 Zu Recht weisen Erich Zenger/Christian Frevel 2012, 406, darauf hin, dass dieser „Tun-Ergehen-Zusammenhang“ nicht als Vergeltungsprinzip verstanden werden will, sondern angemessener von „konnektiver Gerechtigkeit“ gesprochen werden sollte, deren Garant JHWH ist. Vgl. dazu Bernd Janowski 2019, 503-508.

12 Vgl. dazu Bernd Janowski 2019, 148-155 mit weitreichender Literatur. 
ben will, muss für das Wohlbefinden der Tiere, das sich im Aussehen zeigt (vgl. Arnd Meinhold 1991, 463), sorgen (vgl. Magne Sæbø 2011, 335) und achtsam mit den Kleinviehherden umgehen. Auch wenn die Tiere dem Menschen zu Nutze sind, dominiert doch hier das Wissen um die gegenseitige existenzielle Bezogenheit. Wer von Tieren und mit Tieren leben will, geht eine Verantwortung für ihr Wohlergeben ein, für ihr Futter, ihre Pflege, ihre Gesundheit und ihren Schutz. Sonst gehen die Tiere ein. Einseitige Ausbeutung, Vernachlässigung; Tiere als bloße Ressource - das ist dem Weisheitslehrer klar -, führt in den Ruin, zu leeren Vorratskammern, letztlich zu Hunger und Not. Dies ist die elementare und durch Erfahrung geerdete Weisheit bäuerlicher Lebensführung und Lebenserfahrung. Und aus diesen Erfahrungen generiert die alte Weisheit Grundsätze für zukünftiges Handeln: „Kümmere dich sorgfältig ...“.

Eine dezidiert theologische Perspektive ist hier nicht greifbar; nur implizit, aus dem Kontext des Gesamtbuches ist deutlich, dass die Ordnung der Welt die von JHWH selbst gestiftete Ordnung ist. Das sieht in Spr $12,10 \mathrm{a}$, dem - vorgegebenen - Leitmotto des Beitrages, anders aus. Dort heißt es:

Einer, der sich um das Bedürfnis (hebr. næpææ̌) seines Viehs kümmert $\left(j \bar{a} d \bar{a}^{-}\right)$, ist ein Gerechter (hebr. șaddiq).

Anders als Spr 27,23-27 haben wir hier formal keine Ermahnung vor uns, sondern einen sog. Wahrspruch, der thetisch eine gültige Überzeugung formuliert. Wieder steht das Verb jäd $\bar{a}^{\prime}$,kennen, wissen, sich kümmern um“, das schon für Spr 27,23 leitend war, am Beginn. Doch jetzt wird mit $j \bar{a} d \bar{a}{ }^{\prime}$ im Partizip eine kennzeichnende Eigenschaft hervorgehoben: Einer, der sich kümmert ..., ist ein șaddiq, ein Gerechter - wie Noah (vgl. Gen 6,9). Damit tritt in V. 10 ein Leitwort des gesamten Kapitels in den Blick, das acht Mal vorkommt. Der Gerechte wird im Gegensatz zum Frevler (hebr. $r \bar{a} \bar{s}^{-} \bar{a}^{-}$) beschrieben: Der Gerechte ist fest verwurzelt, doch der Frevler hat keinen Bestand (V. 3, vgl. 7.12); während den Frevler das Unglück trifft, bleibt der Gerechte bewahrt (V. 21; vgl. V. 13.2613). Eine Begründung liefert V. 5:

Die Gedanken der Gerechten sind Recht (hebr. mišpāț ), die Überlegungen der Frevler sind Betrug.

13 Die Lesung von V. 26 a ist unklar. Während beispielsweise Bruce K. Waltke 2004, 388, übersetzt: „A righteous man searches out his confidential friend, ...“, schlägt Otto Plöger 1984, 146, eine von MT abweichende Punktation vor und liest: „Es hält Ausschau nach seiner Weide der Gerechte“. 
Der Gerechte wurzelt sozusagen in den rechten Ordnungen der Welt, die von JHWH herkommen. Und wie sich JHWH selbst im Alten Testament als șaddiq in seinem rettenden und heilbringenden Handeln zeigt (vgl. Bo Johnson 1989, 917), so auch der Gerechte, der durch sein rechtes und gerechtes Handeln das Gegenbild des Frevlers ist und das Wohlgefallen JHWHs erlangt (vgl. V. 2). V. 10 führt dies aus: Der Gerechte ist einer, der sich um das Bedürfnis seines Viehs kümmert, um Nahrung, Lebensraum, natürliche Bindung der Tiere untereinander ${ }^{14}$ - in diesem Handeln erweist er sich beispielhaft mit den Ordnungen der Welt in Einklang. Er ist der rechte Weise. Sein Achten bezieht sich zwar, vergleichbar Spr 27, auf das eigene Vieh - es geht nicht um die Tierwelt im allgemeinen, sondern um die

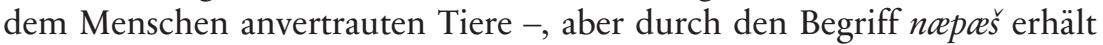
das Mensch-Tier-Verhältnis eine andere, tiefere Verbindung. næep $̌$ hat eine große semantische Breite und bezeichnet Kehle/Schlund/Rachen, aber auch das Verlangen und Bedürfnis; darüber hinaus Lebendigkeit; das vitale Selbst, die Seele? und auch das Lebewesen, die Person (vgl. Horst Seebass 1986). Wird hier mit „Bedürfnis/Bedürftigkeit“ übersetzt, so ist dies dem Kontext angemessen und lenkt auf das „Futter, das durch die Kehle geht“ (Arnd Meinhold 1991, 207); jedoch klingt ebenfalls Lebendigkeit und Lebenskraft mit, die den Tieren innewohnen und sie ausmachen. Doch napaš ist nicht auf Tiere beschränkt, sondern napæes ist einer der anthropologischen Grundbegriffe überhaupt. ${ }^{15}$ Als locus classicus kann hier Gen 2,7 angeführt werden:

Da bildete JHWH Gott den Menschen (aus) Staub vom Erdboden und blies in seine Nase Atem des Lebens; so wurde der Mensch eine lebendige nжра

Die Lebendigkeit kommt von Gott her, sie ist nicht nur den Menschen, sondern eben auch den Tieren verliehen, wie es der priesterliche Schöpfungsbericht $($ Gen 1,30) unterstreicht:

Aber allen Tieren der Erde und allen Vögeln des Himmels und allem, was auf der Erde kriecht, in dem eine lebende næpæ̌̌s/Lebendigkeit ist, alles grüne Kraut (sei ihnen) zur Speise. Und so geschah es.

14 Das zeigt sich in den Rechtsbestimmungen, die untersagen, dass die ,innige Beziehung zwischen Muttertier und säugendem Jungtier“ (Peter Riede 2002 b, 63) sofort zerstört werden darf (Ex 22,29 b; Lev 22,27).

15 Vgl. u. a. Hans Walther Wolff 2010, 33 ff und Bernd Janowski 2014, 73-116. 
Tiere sind von Anfang an Teil der Schöpfungswelt Gottes. napaš rückt diese Mensch und Tier gemeinsame Geschöpflichkeit in den Blick, die der Gerechte erkennt und anerkennt und danach sein Handeln ausrichtet. Walther Pangritz $(1963,86)$ drückt dies so aus:

„Das Tier wird also [...] als beseeltes Mitgeschöpf verstanden, nicht als bloßer Besitz, über den wir verfügen können [...]. Das Wissen um die Seele des Tieres, die Erkenntnis seiner Lebensbedürfnisse und Wünsche verpflichtet den Gerechten, den Menschen, der nach dem Willen Gottes leben möchte, dem Tier, das ihm dient, brüderlich zu helfen, sich seiner zu erbarmen, statt [...] an seinen Lebensbedürfnissen vorüberzugehen“.

Besonders profiliert wird die Handlungsweise des Gerechten in Spr 12,10 a durch die Antithese in V. 10 b:

aber das Innere (hebr. rahamim) des Frevlers ist grausam.

Im Inneren, dem Ort der Eingeweide, sind im Hebräischen die Gefühle, die Zuwendung und Barmherzigkeit lokalisiert. Auffällig ist, dass der Bezugspunkt der Grausamkeit hier offengelassen ist, d. h., dass „(e)ine Einschränkung auf Tiere [...] in diesem Fall nicht vor(liegt)" (Arnd Meinhold 1991, 207). Vielmehr erscheint es dadurch so, dass der Frevler Barmherzigkeit weder gegenüber Menschen noch oder vielleicht schon gar nicht gegenüber Tieren kennt, denn: Grausamkeit zeichnet sein Wesen aus. Die Zuwendung/die Barmherzigkeit des Gerechten dagegen beginnt schon beim Vieh, in dem er das Mitgeschöpf erkennt. ${ }^{16}$

Getragen von dem Wissen der in den Proverbien anzutreffenden existenziellen Verbindung von Mensch und Tier und ihrer Geschöpflichkeit sind auch viele Rechtsbestimmungen, von denen jetzt einige exemplarisch dargestellt werden sollen.

\subsection{Rechtsbestimmungen}

Rechtsbestimmungen in vielen Rechtskorpora, ob Deuteronomium oder Bundesbuch, sind von einem starken Sozialethos durchzogen, das sich

16 Daher wird in manchen Bibel-Übersetzungen Spr 12,10a auch wiedergegeben mit: „Der Gerechte erbarmt sich seines Viehs“ (so die Lutherübersetzung 2017). Meinhold, Spr I, 207, sieht in Spr 12,10 a ein Modell von menschlicher Herrschaft, wie es in Gen 1 gemeint sei, denn im Alten Orient bedeutet Herrschaft Sorge und Schutz für die Untergebenen. 
auch auf das Mensch-Tier-Verhältnis bezieht bzw. die Tiere mit einbezieht. Dies soll an drei Beispielen deutlich werden: In einem apodiktischen Rechtssatz in Dtn 25,4, hinter dem die göttliche Autorität des Sprechers präsent ist, wird gefordert:

Du sollst dem Ochsen nicht das Maul verbinden, wenn er drischt.

Dieses Gebot richtet sich gegen die Gewohnheit, das Zugtier (Ochse oder Rind) „zuzubinden“, d. h. ihm einen Maulkorb umzubinden (vgl. Gustaf Dalman 1933, 98). Denn „(d)en zum Dreschen eingesetzten Tieren wurde das Maul verbunden, damit sie nicht während des Dreschens Getreide fressen, was in doppelter Hinsicht als nachteilig gelten konnte, da sich die Arbeitsleistung der Tiere reduzierte und gleichzeitig das Getreide“ (Eckart Otto 2017, 1849). Hierin wird im Dtn eine unzumutbare Härte gegenüber dem Tier gesehen, das den Dreschschlitten zieht: Ihm soll der Ackerbauer während seiner Arbeit nicht die Nahrung verweigern, sondern ihm seinen Teil zugestehen, d. h. sein Bedürfnis erfüllen. Eine inhaltliche Nähe zu Spr 27 ist hier m. E. greifbar. Die Regelung als Rechtssatz lässt die Vermutung $\mathrm{zu}$, dass dieser Umgang mit dem Arbeitstier jedoch nicht die allgemein verbreitete Norm war.

Während dieser deuteronomische Rechtssatz das Ergehen des Arbeitstiers während der Arbeit im Blick hat, zeugen die folgenden Regelungen aus dem Bundesbuch (und dem Heiligkeitsgesetz) von der Einbindung der Arbeitstiere in den grundlegenden Rhythmus der bäuerlichen Arbeit. Beide Male handelt es sich um Bestimmungen zum bäuerlichen Brachjahr und Ruhetag bzw. Sabbatgebot.

Im Bundesbuch Ex 23,10-12 heißt es:

10 Sechs Jahre sollst du dein Land besäen und seinen Ertrag einsammeln.

11 Aber im siebten sollst du es brachliegen lassen und nicht bestellen, damit die Armen deines Volkes davon essen. Und was sie übrig lassen, soll das Getier des Feldes (hajjat haśädaeh) fressen. Ebenso sollst du es mit deinem Weinberg (und) mit deinem Ölbaum halten.

${ }^{12}$ Sechs Tage sollst du deine Arbeiten verrichten. Aber am siebten Tag sollst du ruhen, damit dein Rind und dein Esel ausruhen und Atem schöpfen der Sohn deiner Magd und der Fremde.

Rainer Albertz hat überzeugend dargelegt, dass hinter diesen Bestimmungen Tabugesetze stehen, die eine menschliche Scheu erkennen lassen, die natürliche Welt vollkommen zu unterwerfen und auszubeuten sowie die Arbeitskraft - auch gerade der Tiere zugunsten der Menschen - völlig auszunutzen (vgl. Rainer Albertz 2015, 10 f). Daher dominiert im Bundesbuch 
die soziale Ausrichtung, die die Tiere ebenfalls mit einbezieht: Die Wildtiere, die sich vom Ertrag der Brachfelder ernähren dürfen ${ }^{17}$ - was bei bestellten Feldern sonst natürlich mit allen Mitteln verhindert wird! - und die domestizierten Tiere Rind und Esel, Zug- und Lasttier, die sich von der Arbeit ausruhen dürfen und der menschlichen Verfügungsgewalt für diese Zeit entzogen sind (vgl. Rainer Albertz 2015, 10 f).

In der exilischen Zeit wird dieser Gedanke von den priesterlichen Bearbeitern zum Sabbatgebot in Ex 20,8-11 umgeformt.

${ }^{8}$ Denke an den Sabbattag, um ihn heilig zu halten.

${ }^{9}$ Sechs Tage sollst du arbeiten und all deine Arbeit tun,

10 aber der siebte Tag ist Sabbat für JHWH, deinen Gott. Nicht sollst du tun alle Arbeit, du und dein Sohn und deine Tochter, dein Knecht und deine Magd und dein Vieh (hebr. $\left.b^{e} h e m a ̄ h\right)$ und der Fremde bei dir, der innerhalb deiner Tore (wohnt).

${ }^{11}$ Denn an sechs Tagen hat JHWH den Himmel und die Erde gemacht, das Meer und alles, was in ihnen ist, und er ruhte am siebten Tag; darum segnete JHWH den Sabbattag und heiligte ihn.

Der siebente Tag bekommt als wöchentliche Institution eine religiöse Qualität als „Sabbat für JHWH“. Mensch und Vieh ${ }^{18}$ (behemāh) „fügten [...] sich einer universalen Ordnung der Zeit ein und transzendierten die vorfindlich geschaffene Welt immer wieder auf den Schöpfer hin“ (Rainer Albertz 2015, 64). D h., auch hier sind Mensch und Tier/Nutztier in der ihnen gemeinsamen Geschöpflichkeit gesehen und in das religiöse Symbolsystem eingeordnet.

Dennoch kann, gerade auch da hier die priesterliche Sicht der Welt zur Sprache kommt, ein letzter Text nicht ausgespart werden, der explizit die Verfügungsgewalt des Menschen über die Tiere thematisiert: die Ermächtigung zur Tiertötung in Gen 9.

17 Vgl. dazu die Anordnung im Heiligkeitsgesetz Lev 25,2-7, wo die Idee des Brachjahres zum allgemeinen Sabbat für das ganze Land stilisiert wird, dessen Sabbatertrag nicht nur den Menschen, sondern auch dem Wildgetier und dem Vieh Nahrung sein soll, vgl. Erhard S. Gerstenberger 1993, 342-344.

18 In der wohl älteren Fassung in Dtn 5,14 wird die Formulierung aus Ex 23,12 aufgegriffen und um das Vieh erweitert: dein Rind und dein Esel und all dein Vieh; die Begründung erinnert an die Befreiung aus Ägypten, so dass die Arbeitsruhe auch hier die Bedeutung von (schöpfungsgemäßer) Freiheit bekommt, vgl. dazu Rainer Albertz 2015, 60-62. 


\section{Menschliche Verfügungsgewalt über die Tiere in Gen 9}

Die priesterschriftliche Neuregelung der Welt nach der Sintflut wird in einer doppelten Gottesrede an Noah und seine Söhne entfaltet. In Gen 9,1-7 heißt es:

${ }^{1}$ Und es segnete Gott Noah und seine Söhne; und er sagte zu ihnen: Seid fruchtbar und werdet zahlreich und füllet die Erde an.

${ }^{2}$ Und Furcht vor euch und Schrecken vor euch sei auf allen Tieren der Erde und auf allen Vögeln des Himmels; mit allem, was sich auf dem Erdboden regt, und allen Fischen des Meeres sind sie in eure Hand (= Verfügungsgewalt) gegeben.

${ }^{3}$ Alles, was sich reget, das lebendig ist: euch sei es zur Speise; wie das grüne Kraut habe ich euch alles gegeben.

${ }^{4}$ Nur Fleisch, als dessen Lebenskraft (= næapaš) sein Blut (vorhanden) ist, sollt ihr nicht essen.

${ }^{5}$ Jedoch euer Blut eurer Lebenskraft (in dem euer jeder Lebenskraft steckt)

will ich einfordern, von jedem Tier will ich es einfordern;

und von dem Menschen, von jedem [seines Bruders], will ich einfordern die Lebenskraft/das Leben (= næpæes $)$ des Menschen.

${ }^{6}$ Derjenige, der Menschenblut vergießt, dessen Blut wird um des Menschen willen vergossen werden; denn als Bild Gottes hat er den Menschen gemacht. ${ }^{7}$ Und ihr: Seid fruchtbar und werdet zahlreich; wimmelt auf der Erde und werdet zahlreich auf ihr.

Der Mehrungssegen (V. 1 und 7) bildet einen Rahmen um den Text Gen 9,2-6 und weist auf die Schöpfungsgeschichte in Gen 1 zurück (vgl. Gen 1,28). Doch Gen 9 geht nicht mehr von der uranfänglichen Schöpfungsordnung aus, sondern ist nur als Reaktion auf die Ursachen der Sintflut, wie sie Gen 6,11-13 nennen, angemessen zu verstehen:

11 Und verderbt wurde die Erde vor Gott, und angefüllt wurde die Erde von Gewalttat.

12 Und Gott sah die Erde und siehe: sie war verderbt, denn verdorben hatte alles Fleisch seinen Weg auf der Erde. 
${ }^{13}$ Und Gott sprach zu Noah:

Das Ende allen Fleisches ist gekommen vor mich, denn angefüllt ist die Erde von Gewalttat durch sie; und siehe ich bin der, der sie verdirbt samt der Erde.

In Gen 6 wird der Einbruch von Gewalttat (hebr. hamas) = Bluttat in die Welt konstatiert (V. 11), und das führt den Schöpfer zur Revision des Urteils von Gen 1,31: Die Welt ist nicht mehr „sehr gut“, sondern „verderbt“ (V. 12), voll mit Gewalttat allen Fleisches - zwischen Mensch und Tier; zwischen Tier und Tier und zwischen Mensch und Mensch. Diese blutigen Übergriffe haben das Schöpfungskonzept von Gen 1 „verdorben“, in dem jedes Lebewesen seinen Ort (Himmel, Meer und Land/Erde) und seine vegetabile (!) Nahrung (Gen 1,29f) zugewiesen bekommen hatte: die Menschen die samentragenden Kräuter, sprich: Gemüse, und Bäume, deren Früchte Samen tragen, die Tiere dagegen Kraut der Pflanze, Blattgrün, das die Erde ohne Kultivierung hervorbringt.

Die Rede an Noah nach der Sintflut knüpft zwar an Gen 1 an, indem es die Ausbreitung der Noachiten als Beginn einer neuen Menschheit mit Segen verheißt - den Tieren war schon in Gen 8,15 der Mehrungssegen zuteil geworden -, doch die ursprüngliche Unschuld des Mensch-Tier-Verhältnisses ist verloren. Die Neuregelungen in Gen 9 ordnen sich der Realität der Gewalttat unter - der gegenwärtig erlebbaren Realität seiner Adressaten. Sie sind einseitige Regelungen für den Menschen und zu seinem Schutz.

„Furcht und Schrecken“ soll auf den Tieren lasten (V. 2), eine Redeweise, die in deuteronomisch/deuteronomistisch geprägten Texten Israels Überlegenheit gegenüber den Feinden herausstellt und als Ermutigung zum Kampf fungiert, weil die von Gott gewirkten Furcht und Schrecken die Feinde lähmen und wehrlos machen. Übertragen auf das Verhältnis Mensch-Tierwelt wird die unantastbare Überlegenheit des Menschen festgeschrieben. Gen 9,2 ist getragen von dem Gedanken, die Gewalttat von Tieren gegen den Menschen zu unterbinden. Diese heute als anthropozentrisch stigmatisierte Sicht zeugt davon, „wie gravierend die Priesterschrift die Kluft zwischen den Menschen und seinen Mitgeschöpfen empfunden hat und wie konfliktträchtig das Zusammenleben von Mensch und Tier in dem gemeinsamen Lebensraum "Erde" in Wirklichkeit ist" (Bernd Janowski 1999, 44).

Gleichzeitig wird dem Menschen Verfügungsgewalt über die Tierwelt von Gott eingeräumt (V. 2): Das Töten von Tieren wird dem Menschen erlaubt (V. 3). Damit ist die Grenzziehung zwischen den Menschen und den Tieren, die in Gen 1,29f durch die unterschiedliche vegetabile Nahrung 
aufgerichtet war, durchbrochen. Die menschliche Herrscherposition wird einseitig zuungunsten der Tierwelt verstärkt. Aber ein blindwütiges Töten, Gewalt- und Bluttat wie in Gen 6 ist nicht gemeint. Die Zweckbindung „zur Speise“ erweist die nüchtern erteilte Tötungserlaubnis nicht als Bluttat, die den Bestand der Schöpfung gefährdet, sondern als begrenzt zugestandene Gewalt. Tötung von Tieren ist erlaubt, aber nicht Ausrottung oder Töten aus Lust, nicht zügellose Gewalttat. Auch V. 4 wehrt einem ungehemmten Blutrausch, denn das Blut als Sitz der Lebenskraft (næ⿻ жš́) wird dem menschlichen Verzehr entzogen, es bleibt als Sühnemittel dem Altar/Gott vorbehalten. ${ }^{19}$ Die Verfügungsgewalt des Menschen endet da, wo sich die lebensspendende Kraft des Schöpfergottes zeigt.

Die Erlaubnis der Tiertötung ist das einseitige Zugeständnis von menschlicher Gewalt gegen Tiere und geht mit dem unbedingten Schutz menschlichen Lebens (V. 5 f) einher. „Die Erlaubnis, Fleisch zu essen, aber nicht mit seinem Lebensblut, und die Warnung, Menschenblut zu vergießen, normieren die grundsätzliche Stellung des Menschen innerhalb der gesamten Lebenswelt, das Verhältnis zu seinesgleichen und zum Tier" (Benno Jacob 1934/2000, 250).

Doch obwohl die Priesterschrift die Vorherrschaft des Menschen und seine Integrität betont, sichert sie der Tierwelt in ihrer gesamten Ausprägung ein Lebensrecht $\mathrm{zu}$ - wie schon am Sabbatgebot zu sehen war. So bindet die zweite Gottesrede an Noah und seine Söhne die Tiere in den Gottesbund mit ein (Gen 9,8-11):

${ }^{8}$ Dann sprach Gott zu Noah und zu seinen Söhnen, die bei ihm waren:

${ }^{9}$ Ich aber, ich richte meinen Bund auf mit euch und mit euren Nachkommen

10 und mit allen Lebewesen, die bei euch sind, mit den Vögeln, dem Vieh und allen Wildtieren bei euch, mit allem, was aus der Arche gekommen ist, mit allen Tieren der Erde.

11 Ich will meinen Bund mit euch aufrichten: Nie wieder soll alles Fleisch vom Wasser der Sintflut ausgerottet werden, und nie wieder soll eine Sintflut kommen, um die Erde zu verderben.

19 Vgl. dazu Lev 17,11 f: Denn das Leben des Fleisches ist das Blut, und ich habe es euch für den Altar gegeben, damit Sühne für euch erwirkt werden kann. Denn das Blut ist die Lebenskraft und erwirkt Sühne. Darum habe ich zu den Israeliten gesagt: Niemand von euch darf Blut verzehren. Auch der Fremde, der in eurer Mitte lebt, darf kein Blut verzehren. Vgl. dazu u. a. Bernd Janowski/Ute Neumann-Gorsolke 1993, 240-244 und Ute Neumann-Gorsolke 2016, 57-60. 
Gen 9,8-11 widerrufen das Tötungsrecht des Menschen nicht, aber machen deutlich, dass auch Tiere als Bundespartner zu Gottes Schöpfung gehören. Von diesem Bund sind Mensch und Tier als Geschöpfe umschlossen, d. h. den Tieren kommt ein Lebensrecht und ein Eigenwert in der göttlichen Schöpfung zu, die über eine allein auf den Menschen ausgerichtete Verwertung deutlich hinausgehen.

Nirgends wird diese Haltung fassbarer als im Schöpferpsalm 104 und in den Gottesreden des Ijobbuches (Kap. 39 f):

5 Wer hat den Wildesel freigelassen, / des wilden Esels Fesseln, wer schloss sie auf?

${ }^{6}$ Ich gab ihm zur Behausung die Steppe, / zu seiner Wohnung die salzige Trift.

${ }^{7}$ Er verlacht das Lärmen der Stadt, / hört nicht des Treibers Geschrei.

${ }^{8}$ Die Berge sucht er nach Weide ab, / jeglichem Grün spürt er nach.

9 Wird dir der Wildstier dienen wollen, / bleibt er an deiner Krippe zur Nacht?

${ }^{10}$ Hältst du am Seil ihn in der Furche, / pflügt er die Täler hinter dir her?

11 Traust du ihm, weil er so stark ist? / Überlässt du ihm deine Arbeit?

12 Glaubst du ihm, dass er wiederkommt / und deine Saat auf die Tenne bringt?

(Ijob 39,5-12 Einheitsübersetzung)

Gott hält dem leidenden und ihn anklagenden Hiob die Größe seiner Schöpfermacht und die Vielfalt der Schöpfung vor Augen, vor der der Mensch Hiob nur klein und unbedeutend erscheinen kann. Die Gottesrede hebt die Freiheit und Ungebundenheit der Wildtiere hervor, die auch ohne den Menschen existieren, die der Mensch weder zu zähmen noch zu beherrschen vermag. Sie alle haben, auch ohne dass sie von Nutzen für die Menschen sind, ein vom Schöpfer verbürgtes Lebensrecht, das ihnen der Mensch nicht nehmen kann.

\section{Kurzes Resümee}

- Was wir aus den Schriften des Alten Testaments erfahren und was von der Ikonographie und Archäologie bekräftigt wird, ist, dass das Verhältnis von Mensch und Tier im alten Israel/bei den Israeliten ein unmittelbares war: Tiere prägten das Alltagsleben, lebten mit unter einem Dach und bildeten die Basis des wirtschaftlichen Lebens. 
- Hieraus ergibt sich - wie die Texte der älteren Weisheit zeigen - eine aus der Erfahrung gewonnene Wertschätzung der Haustiere und zugleich die Erkenntnis, dass die Sorge und Pflege und auch der Schutz der Herdentiere die eigene Existenz sichern, Vernachlässigung und Ausbeutung jedoch Schaden oder Ruin für den Menschen bedeuten kann. Eine existentielle Bezogenheit prägt das Mensch-Tier-Verhältnis.

- In den Geboten des Deuteronomiums und des Bundesbuches wird deren sozialethische Perspektive auf Tiere ausgedehnt: Der dreschende Ochse soll Anteil an der Ernte erhalten, die Zug- und Lasttiere werden in die allgemeine Arbeitsruhe am 7. Tag einbezogen; auch die spätere exilische Sabbattradition der Priesterschrift hält an dieser Einbeziehung der Tiere in der vom Schöpfer vorgegebenen Rhythmik des Lebens fest. Hierin zeigt sich, wie eng verknüpft Tiere mit dem menschlichen Leben waren.

- Allen Überlieferungen ist implizit oder explizit gemein, dass Mensch und Tier beide als „lebendige nжрқs" zu verstehen sind, die ihre Lebenskraft vom Schöpfer erhalten (vgl. Spr 12,10; Gen 2,7; Gen 1,30 u. ä.). Sie sind beide Geschöpfe Gottes und haben dieselben Grundbedürfnisse nach Nahrung, Pflege und Schutz, und sie ereilt dasselbe Ende, der Tod (vgl. Koh 3,19). Wie der Mensch haben auch Tiere als Geschöpfe Gottes ein Lebensrecht, ja ein Eigenrecht auf Leben auch jenseits menschlichen Nutzens (vgl. Ijob 39).

- Von einer heute diskutierten egalitaristischen Ethik sprechen die alttestamentlichen Texte jedoch nicht: Tiere sind dem Menschen zu Nutze und sichern durch Arbeit, Milch, Fell und Fleisch ihr Leben. Wie wir darüber hinaus in Gen 9,2-6 lesen, ist der primäre Schutz der Menschen einerseits und seine Verfügungsgewalt über Tiere für seine Nahrung andererseits in einer Welt, die von der Realität der Gewalttat unter den Geschöpfen und der Bedrohung des Menschen durch wilde Tiere bestimmt wird, für die exilische Priesterschrift unabdingbar, allerdings ohne Ausbeutung oder Ausrottung von Tieren das Wort zu reden. Denn auch in diesem Text wird deutlich, dass die Lebenskraft von Gott kommt und die Tiere zum Bund Gottes mit der Welt gehören. Die Ambivalenz des Tier-Mensch-Verhältnisses in der Welt löst der Text nicht auf.

- Dass eine Welt ohne Tiere für die biblischen Schriftsteller/Menschen undenkbar war, wird auch an den eschatologischen Vorstellungen im Jesajabuch greifbar, die als Tierfrieden eine Welt ohne Gewalt unter allen Geschöpfen evozieren, eine Welt, wo Wolf und Lamm einträchtig weiden werden (Jes 65,25). 
- Doch bis dahin sind wir vor die Aufgabe gestellt, in Erinnerung und in Auseinandersetzung mit den biblischen Texten unser Tierverhältnis zu überdenken und herauszufinden, was das Bedürfnis der Tierwelt ist, die uns anvertraut ist - auch damit wir selbst leben!

\section{Literatur}

Albertz, Rainer 2015, Exodus 19-40 (Zürcher Bibelkommentar Altes Testament), Zürich.

Bergman, Jan/Botterweck, Gerhard J. 1982, Art. ידע, jädā', in: Theologisches Wörterbuch zum Alten Testament III, 479-512.

Bunimovitz, Shlomo/Faust, Avraham 2009, Building Identity. Das Vierraumhaus und der „Israelite Mind“, in: Janowski, Bernd/Liess, Kathrin (Hg.) 2009, Der Mensch im alten Israel. Neue Forschungen zur alttestamentlichen Anthropologie, Freiburg etc., 401-418.

Dalman, Gustaf 1933, Arbeit und Sitte in Palästina, Bd. III: Von der Ernte zum Mehl. Ernten, Dreschen, Worfeln, Sieben, Verwahren, Mahlen, 2. Nachdruckauflage 1987, Hildesheim etc.

Gerstenberger, Erhard S. 1993, Das dritte Buch Mose. Leviticus (Das Alte Testament Deutsch 6), Göttingen.

Jacob, Benno 1934/2000, Das Buch Genesis, Stuttgart.

Janowski, Bernd 1999, Auch Tiere gehören zum Gottesbund. Gott, Mensch und Tier im alten Israel, in: Janowski, Bernd/Riede, Peter 1999, Die Zukunft der Tiere. Theologische, ethische und naturwissenschaftliche Perspektiven, Stuttgart, 31-60.

Janowski, Bernd 2014, Die lebendige næpæš. Das Alte Testament und die Frage nach der „Seele“, in: Janowski, Bernd 2014, Der nahe und der ferne Gott (Beiträge zur Theologie des Alten Testaments 5), Neukirchen-Vluyn, 73-116.

Janowski, Bernd 2019, Anthropologie des Alten Testaments. Grundfragen - Kontexte - Themenfelder, Tübingen.

Janowski, Bernd/Neumann-Gorsolke, Ute 1993, Opfertiere und Tieropfer, in: Janowski, Bernd/Neumann-Gorsolke, Ute/Gleßmer, Uwe (Hg.) 1993, Gefährten und Feinde des Menschen. Das Tier in der Lebenswelt des alten Israel, Neukirchen-Vluyn, 240-244.

Keel, Othmar 1993, Allgegenwärtige Tiere. Einige Weisen ihrer Wahrnehmung in der hebräischen Bibel, in: Janowski, Bernd/Neumann-Gorsolke, Ute/Gleßmer, Uwe (Hg.) 1993, Gefährten und Feinde des Menschen. Das Tier in der Lebenswelt des alten Israel, Neukirchen-Vluyn, 155-193.

Keel, Othmar/Küchler, Max/Uehlinger, Christoph 1984, Orte und Landschaften der Bibel. Ein Handbuch und Studienreiseführer zum Heiligen Land, Bd. 1: Geographisch-geschichtliche Landeskunde, Zürich etc.

Kessler, Rainer 2017, Der Weg zum Leben. Ethik des Alten Testaments, Gütersloh. 
Meinhold, Arnd 1991, Die Sprüche, Teil II: 16-31 (Zürcher Bibelkommentar Altes Testament), Zürich.

Neumann-Gorsolke, Ute 2016, „In eure Hand sind sie gegeben ...“ (Gen 9,2). Tiertötung im Alten Testament, in: Joachimides, Alexis/Milling, Stephanie/Müllner, Ilse/Thöne, Yvonne Sophie (Hg.) 2016, Opfer - Beute - Hauptgericht. Tiertötungen im interdisziplinären Diskurs (Human Animal Studies), Bielefeld, 47-67.

Otto, Eckart 2017, Deuteronomium 12-34, Zweiter Teilband: 23,16-34,12 (Herders Theologischer Kommentar zum Alten Testament), Freiburg etc.

Pangritz, Walther 1963, Das Tier in der Bibel, München.

Plöger, Otto 1984, Sprüche Salomos. Proverbia (Biblischer Kommentar Altes Testament XVII), Neukirchen-Vluyn.

Riede, Peter 2000, Im Netz des Jägers. Studien zur Feindmetaphorik der Individualpsalmen (Wissenschaftliche Monographien zum Alten und Neuen Testament 85), Neukirchen-Vluyn.

Riede, Peter $2002 \mathrm{a}$, Im Spiegel der Tiere. Überlegungen zum Verhältnis von Mensch und Tier in der christlich-jüdischen Tradition, in: Riede, Peter 2002, Im Spiegel der Tiere. Studien zum Verhältnis von Mensch und Tier im alten Israel (Orbis Biblicus Orientalis 187), Freiburg (Schweiz)/Göttingen, 29-56.

Riede, Peter 2002 b, Der Gerechte kennt die Bedürfnisse seiner Tiere. Der Mensch und die Haustiere in der Sicht des Alten Testaments, in: Riede, Peter 2002, Im Spiegel der Tiere. Studien zum Verhältnis von Mensch und Tier im alten Israel (Orbis Biblicus Orientalis 187), Freiburg (Schweiz)/Göttingen, 57-64.

Riede, Peter 2010, Art. Löwe, in: Wissenschaftliches Lexikon zur Bibel (wibilex.de), https:/www.bibelwissenschaft.de/stichwort/25081/ (abgerufen am 04.02.2020).

Ringgren, Helmer/Johnson, Bo 1989, Art. צדק/șädaq, in: Theologisches Wörterbuch zum Alten Testament VI, 898-924.

Sæbø, Magne 2011, Sprüche (Das Alte Testament Deutsch 16,1), Göttingen.

Schmitz, Friederike 2014, Tierethik. Eine Einführung, in: Schmitz, Friederike (Hg.) 2014, Tierethik. Grundlagentexte (stw), Berlin, 13-76.

Seebass, Horst 1986, Art. נפש/napaš, in: Theologisches Wörterbuch zum Alten Testament V, 531-555.

Thomsen, Iris 2012, Flusspferde am See Gennezareth, in: Welt und Umwelt der Bibel 63, 60-61.

Waltke, Bruce K. 2004, The Book of Proverbs, Ch 1-15 (The New International Commentary on the Old Testament), Grand Rapids, MI/Cambridge, UK.

Wolff, Hans Walther 2010, Anthropologie des Alten Testaments (1973). Mit zwei Anhängen neu herausgegeben von Bernd Janowski, Gütersloh.

Zenger, Erich/Frevel, Christian 2012, Eigenart und Bedeutung der Weisheit Israels, in: Zenger, Erich/Frevel, Christian (Hg.) ${ }^{8} 2012$, Einleitung in das Alte Testament (Kohlhammer Studienbücher Theologie 1,1), Stuttgart, 405-413. 


\section{Abbildungsnachweis}

Abb. 1: Keel, Othmar/Uehlinger, Christoph ${ }^{4} 1998$, Göttinnen, Götter und Gottessymbole. Neue Erkenntnisse zur Religionsgeschichte Kanaans und Israels aufgrund bislang unerschlossener ikonographischer Quellen (Quaestiones Disputatae 134), Freiburg i. Br. etc., 209.

Abb. 2: Calwer Bibellexikon ${ }^{6} 1989$, Stuttgart, Sp. 1246.

Abb. 3: Zwickel, Wolfgang 1997, Die Welt des Alten und Neuen Testaments. Ein Sach- und Arbeitsbuch, Stuttgart, 119, Abb. 15.

Abb. 4: Vieweger, Dieter 2003, Archäologie der biblischen Welt, Göttingen, 46, Abb. 34.

Abb. 5: Zwickel, Wolfgang 1997, Die Welt des Alten und Neuen Testaments. Ein Sach- und Arbeitsbuch, Stuttgart, 155, Abb. 57. 